\title{
The Prevalence of Celiac Disease in Patients with Diabetes Mellitus
}

\author{
Deniz Gökalp', Senay Arıkan', Mustafa Yakut ${ }^{2}$, Yekta Tüzün³ ${ }^{3}$, Alparslan Kemal Tuzcu', Mithat \\ Bahçeci $^{1}$, Senay Arikan ${ }^{1}$, İrfan Soykan ${ }^{2 *}$
}

\author{
${ }^{1}$ Endocrinology, Dicle University Medical School, Diyarbakır, Turkey; ${ }^{2}$ Gastroenterology Ankara University Medical School, An- \\ kara, Turkey; ${ }^{3}$ Gastroenterology, Dicle University Medical School, Diyarbakır, Turkey. \\ Email: "isoykan@medicine.ankara.edu.tr
}

Received December 19 ${ }^{\text {th }}, 2010$; revised April 6 ${ }^{\text {th }}, 2011$; accepted April 26 ${ }^{\text {th }}, 2011$.

\begin{abstract}
Aim: To determine the prevalence of celiac disease related autoantibodies in patients with type 1 and 2 diabetes mellitus (DM), and to compare these results with the general population. Methods: In total, 137 consecutive patients with type 1 DM, 172 with type $2 D M$ and 113 age-sex matched control subjects were included into the study. Antigliadin-autoantibodies (AGA) IgG and IgA, and endomysial-antibodies (EMA) IgG and IgA antibodies were determined. Patients who were positive for one or more were offered a gastroduodenoscopic examination. Results: AGA IgG positivity was detected in $38.7 \%$ (53/137) patients with type 1 DM in $26.2 \%$ (45/172) patients with type 2 DM and in $16.8 \%$ (19/113) control subjects (significant differences). AGA IgA positivity was detected in $24.8 \%$ (34/137) patients with type $1 D M$, in 9.3\% (16/172) patients with type $2 D M$ and in 3.5\% (4/113) control subjects (significant differences). EMA IgG positivity was detected in 10.2\% (14/137) patients with type 1 DM in $0.6 \%(1 / 176)$ patients with type 2 DM and $0.9 \%$ (1/113) control subjects (significant differences). EMA IgA positivity was detected in 11.7\% (16/137) patients with type 1 DM in $0.6 \%$ (1/172) patients with type 2 DM and in none of control subjects. EMA IgA positivity was sig- nificantly higher in patients with type 1 DM as compared with patients with type 2 DM and controls. Conclusions: High prevalence of celiac disease at the diagnosis of type 1 DM is observed. Serological markers are useful for identi- fying celiac disease patients with type 1 DM.
\end{abstract}

Keywords: Celiac Disease, Diabetes Mellitus, AGA IgA, AGA IgG, EMA IgA, EMA IgG

\section{Introduction}

Celiac disease (CD) is proved to be a T-cell mediated gluten intolerance in genetically predisposed individuals and it is made distinct and recognizable by villus atrophy and malabsorption of small intestine. In some people who are genetically apt to $\mathrm{CD}$, it becomes visible after ingestion of foods containing gluten and related proteins (Alaedini and Green, 2005). CD shows a noticeable regional frequence. Western Europe is the region where CD is known to be prevalent. That CD is widespread with an incidence of nearly $1 \%$ [1], has been unveiled by serological screening studies .

It has been confirmed that in patients with type $1 \mathrm{DM}$ has the high ratio of general existence of CD. This existence of relation is declared as $2.3 \%$ to $7 \%$ [2] which are higher than general population. Originally, both of the diseases are known as autoimmune and as a result of an interaction between genetic and environmental factors, they could be obvious. However, in patients with type 2 DM, CD related autoantibodies are not so common [3,4].

Short stature, sideropenic anemia, or hypertransaminasemia are several symptoms which are non-classical forms of the disease that most patients who have diabetes and $\mathrm{CD}$. In many cases, patients are completely symptom-free [5]. The risk of ever-evolving autoimmune diseases, malignancy, osteoporosis, infertility, and intestinal lymphomas is proportional to the time of exposure to gluten in CD patients [6,7]; thus, for the early diagnosis of silent celiac disease, using serologic screening tests is vital. AGA has merely $80 \%$ sensitivity and specificity. [8] EMA has been proved to be a more trustworthy marker than AGA since it has $90 \%$ of sensitivity and specificity [10]. In screening purposes for CD in diabetic patients, the united detection of antigliadin and antiendomysium antibodies is being used because its a sensitivity and specificity is $100 \%$. In order to uncover silent celiac disease in diabetic patients, these 
antibodies are a reliable method.

As we scanned the literature, we did not encounter any data about the frequency of CD related autoantibodies in patients with DM in the Southeastern population of Turkey. For this reason, in this study, we evaluated the prevalence of $C D$ related autoantibodies in patients with type 1 and 2 DM and found a higher frequency of AGA IgA, IgG and EMA IgA, IgG positivity in patients with DM compared with the general population.

\section{Materials and Methods}

The study was performed in Dicle University, Department of Endocrinology between January 2002 and December 2008. In total, consecutive 137 patients with type 1 DM, 172 patients with type 2 DM and 113 age-sex matched control subjects were included into the study.

The laboratory evaluations of all patients and control subjects were performed according to standart methods at Dicle University Medicine Faculty Laboratory services. AGA IgG and IgA, and EMA IgG and IgA antibodies were determined by immunofluorescence method with Euroimmun immunofluorescence autoantibody determination kits. Total IgA level was detected by nefelometric technique by Beckmancoulter image to be able to exclude patients or control subjects with IgA deficiency. Spesific questions were asked and laboratory examinations were performed by same physician to define signs or symptoms of CD; development of diarrhea, hypogonadism, anemia, weight loss, osteoporosis, dermatitis herpetiformis and elevation of transaminases.

Patients who were positive for (AGA Ig A, EMA IgG, and EMA IgA) one or more were offered a gastroduodenoscopic examination. In the intestinal histopathologycal analysis, more than three biopsy specimens were taken from the second part of duodenum during gastroduodenoscopy. Hematoxylineosin staining was used in these specimens. Slides were graded by conventional histology as normal, with partial villous atrophy, and with subtotal villous atrophy.

\section{Statistical Analysis}

Mean value, standard deviation (SD), minimum and maximum values were calculated. Independent $t$ test was used to determine the mean values of the variables. The relation of categorical variables was analyzed with the Chi-square test. A $p$ value $<0.05$ was accepted as statistically significant.

\section{Results}

In total 137 patients with type $1 \mathrm{DM}, 172$ patients with type $2 \mathrm{DM}$ and 113 healthy subjects were included into the study. Out of 137 patients with type $1 \mathrm{DM}, 75$ were female and 62 were male, and out of 172 patients with type $2 \mathrm{DM}, 88$ were female and 84 were male. Out of 113 control subjects, 62 were female and 51 were male. Median age of patients with of type 1 DM was 28 (ranged from 16 to 39), median age of patients with type 2 DM was 42 (ranged from 29 to 68) and the control subjects was 39 (ranged from 29 to 48). There was no statistically significant difference between patients with type $1 \mathrm{DM}$, type $2 \mathrm{DM}$ and control subjects. Median age of patients with type $1 \mathrm{DM}$ was lower than patients with type $2 \mathrm{DM}$ and control subjects $(p<0.0001)$. There is no statistically significant difference between median age of patients with type $2 \mathrm{DM}$ and control subjects. None of the patients and control subjects has IgA deficiency.

AGA IgG positivity was detected in 53 out of 137 (38.7\%) patients with type $1 \mathrm{DM}$, in 45 out of 172 (26.2\%) patients with type $2 \mathrm{DM}$ and in 19 out of 113 (16.8\%) control subjects. There was a statistically significant difference in terms of AGA IgG positivity between patients with type $1 \mathrm{DM}$ and control subjects, and patients with type $1 \mathrm{DM}$ and type $2 \mathrm{DM}$ patients $(p=$ 0.000 and $p=0.019$, respectively). However, there was no statistically significant difference in terms of AGA IgG positivity between patients with type $2 \mathrm{DM}$ and control subjects.

AGA IgA positivity was detected in 34 out of 137 (24.8\%) patients with type $1 \mathrm{DM}$, in 16 out of 172 (9.3\%) patients with type $2 \mathrm{DM}$, and in 4 out of 113 (3.5\%) control subjects.

There was a statistically significant difference in terms of AGA IgA positivity between patients with type $1 \mathrm{DM}$ and control subjects $(p=0.000)$, There was no statisticcally significant difference in patients with type $2 \mathrm{DM}$ as compared with controls. There was a statistically signifycant difference in terms of AGA IgA positivity between patients with type $1 \mathrm{DM}$ and type $2 \mathrm{DM}(p=0.000)$,

EMA IgG positivity was detected in 14 out of 137 (10.2\%) patients with type $1 \mathrm{DM}$, in 1 out of $176(0.6 \%)$ patients with type $2 \mathrm{DM}$ and 1 out of 113 (0.9\%) control subjects. EMA IgG positivity was statistically signifycantly higher in patients with type 1 compared with controls subjects and patients with types $2 \mathrm{DM}(p=0.002, p$ $=0.000$ ). There was no statistically significant difference between types $2 \mathrm{DM}$ and control subjects in terms of EMA IgG positivity.

EMA IgA positivity was detected in 16 out of 137 (11.7\%) patients with type $1 \mathrm{DM}$, in 1 out of $172(0.6 \%)$ patients with type $2 \mathrm{DM}$ and in none of control subjects. EMA IgA positivity was statistically significantly higher in patients with type $1 \mathrm{DM}$ as compared with patients 
with DM type 2 and controls ( $p=0.000, p=0.000$ ). There was no statistically significant difference in terms of EMA IgA positivity of patients between with type 2 DM and controls subjects. AGA-IgA and EMA-IgA positivity was found in 10 out 137 patients with typa 1 $\mathrm{DM}$, in out of 172 patients with type $2 \mathrm{DM}$ and in none of control subjects.Charasteristics of patients with type 1 DM, type $2 \mathrm{DM}$ and control subjects were shown in Table 1.

Of the 309 patients and 113 controls, 55 (13.4\%) were positive for at least one of the three antibodies (AGA IgA, EMA IgG, and EMA IgA), and 41 accepted to undergo an endoscopy. Sixteen patients $10.9 \%$ of the DM1 patients, $0.6 \%$ ( $n=1$ of DM2 patients were histologically diagnosed with CD. Most of these patients had no symptoms of CD.

\section{Discussion}

CD accounts for one of the best-understood examples of an environmentally activated autoimmune disease, with genetic risk factors (HLA-DQ2/DQ8), environmental triggers gliadin, and autoantigenic targets tissue transglutaminase being well defined [11]. Villous atrophy and crypt hyperplasia, which ranges from silent asymptomatic forms to active malabsorption syndromes are the abnormalties in the small intestinal mucosa that show us $\mathrm{CD}$ is a heterogenous disorder. Among patients with celiac sprue, a large number of diseases such as Dermatitis herpetiformis, type $1 \mathrm{DM}$, autoimmune thyroid disease happen to be more frequent.

In patients with type $1 \mathrm{DM}$, its clinical and pathologic manifestations, and typical gastrointestinal symptoms are rare [12]. That screening is necessary to detect $C D$ in patients with type $1 \mathrm{DM}$ is suggested by the increasing numbers of reports of milder, less symptomatic forms of CD without overt signs of malabsorption.

Undoubtedly, modern serology has become a reliable method for better targeting patients to biopsy although small intestinal biopsy has been the value test for diagnosis [13] . In order to screen high risk groups and the general population, both IgG and IgA AGA anti-bodies have been extensively used. They are cheap, easy to measure, and have reasonably high sensitivity

Table 1. Characteristics of patients and control subjects.

\begin{tabular}{llll}
\hline & $\begin{array}{l}\text { DM type 1 } \\
(n=137)\end{array}$ & $\begin{array}{l}\text { DM type 2 } \\
(n=172)\end{array}$ & $\begin{array}{l}\text { Controls } \\
(n=113)\end{array}$ \\
\hline Female/male & $66 / 62$ & $88 / 84$ & $62 / 51$ \\
Median age (year) & $28(16-39)$ & $42(29-68)$ & $39(29-48)$ \\
AGA IgG positive & $53(38.7 \%)$ & $45(26.2 \%)$ & $19(16.8 \%)$ \\
IgA positive & $34(24.8 \%)$ & $16(9.3 \%)$ & $4(3.5 \%)$ \\
EMA IgG positive & $14(10.2 \%)$ & $1(0.6 \%)$ & $1(0.9 \%)$ \\
IgA positive & $16(11.7 \%)$ & $1(0.6 \%)$ & 0 \\
\hline
\end{tabular}

and specificity [14]. On the other hand, the positive foretelling value of this test is low for population screening [15]. In contrast, with high predictive value [15,16], EMA IgA is a highly sensitive (93\% - 98\%) and specific $(99 \%$ - 100\%) test. Some investigators suggested that in the presence of a positive EMA, a duodenal biopsy is not necessary for diagnosis by the use of this high specificity [17]. Since the majority of reports indicating approximately $90 \%$ sensitivity, we understand that generally the sensitivity of the EMA is excellent [18]. The degree of mucosal damage is correlated with the titer of EMA [19].

Two recent screening programs for $\mathrm{CD}$ in a cohort of American type $1 \mathrm{DM}$ patients, found a prevalence of 6.4\% [20,21], just as the same with European patients [22]. In an adult Turkish population with type $1 \mathrm{DM}$, the prevalence of CD is reported as $2.45 \%$ [23]. When we compare Turkish type 1 diabetes mellitus patients with American and European patients, the high prevalence of EMA antibodies (EMA IgG positivity 10.2\%, EMA IgA positivity $11.7 \%$ ) may be caused by excessive bread consumption in our region and the prevalence of gastrointestinal infection in children.

It is suggested by the previous studies that an independent trigger effect on the development of CD might be implemented by type 1 diabetes [24] The reaction to gliadin in susceptible individuals could be triggered by immunoregulatory disturbances in type $1 \mathrm{DM}$. Both type $1 \mathrm{DM}$ and $\mathrm{CD}$ are associated with certain HLA alleles of the DQB1 and DQA1 locus. Some of the co-existance of the two diseases may be explained by the HLA alleles; however, the reason why type $1 \mathrm{DM}$ diagnosis precedes CD diagnosis is still unknown. It has been assumed that there may be a nonspecific activation of the immune system directed toward several dietary proteins attributable to defective immunoregulation or loss of immunologic tolerance of a variety of ingested antigens in patients newly diagnosed with type 1 DM [25].

We found a high prevalence of CD at the diagnosis of type $1 \mathrm{DM}$ in the region. It is important to treat subclinical CD, because patients with undiagnosed CD are at risk of long-term CD complications, such as infertility, anemia, osteoporosis, and malignancy. It has been revealed that untreated celiac disease is associated with high morbidity and increased mortality [25,26]. Although the presentation of patients with celiac disease may be protean, serological markers are a cheap and non-invasive method for clinicians in primary care and secondary care to identify patients with this disease.

\section{REFERENCES}

[1] R. J. Farrell and C. P. Kelly, "Celiac Sprue and Refrac- 
tory,” In: M. Feldman, L. Lawrence and S. Friedman, Eds., Sleisenger and Fordtran's Gastrointestinal and Liver Disease: Pathophysiology, Diagnosis, Management, 2-Volumes Set, Saunders, Philadelphia, 2006, pp. 2277-2306

[2] M. Dirks, "Preventive Health Care, 2004 Update: Screening Persons with Insulin-Dependent Diabetes Mellituswith Serology for Celiac Disease: Systematic Review \& Recommendations,” Canadian Medical Association Journal, In press.

[3] J. C. Cruz, E. C. Rode, L. S. Gomez, et al., "Celiac Disease Associated Antibodies in Persons with Latent Autoimmune Diabetes of Adult and Type 2 Diabetes,” Autoimmunity, Vol. 40, No. 2, 2007, pp. 103-107. doi:10.1080/08916930601118825

[4] C. Catassi, E. Fabiani, I. M. Rätsch, G. V. Coppa, P. L. Giorgi, et al., "The Coeliac Iceberg in Italy. A Multicentre Antigliadin Antibodies Screening for Coeliac Disease in School-Age Subjects," Acta Paediatrica, Vol. 85, No. S412, 1996, pp. 29-35. doi:10.1111/j.1651-2227.1996.tb14244.x

[5] I. De Vitis, G. Ghirlanda and G. Gasbarrini, "Prevalence of Coeliac Disease in Type I Diabetes: A Multicentre Study," Acta Paediatrica, Vol. 85, No. S412, 1996, pp. 56-57. doi:10.1111/j.1651-2227.1996.tb14253.x

[6] W. Dieterich, T. Ehnis, M. Bauer, et al., "Identification of Tissue Transglutaminase as the Autoantigen of Celiac Disease,” Nature Medicine, Vol. 3, 1997, pp. 797-801. doi:10.1038/nm0797-797

[7] R. F. A. Logan, E. A. Rifkind, I. D. Turner and A. Ferguson, "Mortality in Celiac Disease," Gastroenterology, Vol. 97, No. 2, 1989, pp. 265-271.

[8] A. Lerner and E. Lebenthal, "The Controversy of the Use of Anti-Gluten Antibody (AGA) as a Diagnostic Tool in Celiac Disease," Journal of Pediatrc Gastroenterology and Nutrition, Vol. 12, No. 4, 1991, pp. 407-409. doi:10.1097/00005176-199105000-00001

[9] H. Ascher, M. Hahn-Zoric, L. A. Hansson, A. F. Kilander, L. A. Nilsson and H. T. laskalova, "Value of Serologic Markers for Clinical Diagnosis and Population Studies of Coeliac Disease," Scandinavian Journal of Gastroenterology, Vol. 31, No. 1, 1996, pp. 61-67. doi:10.3109/00365529609031628

[10] A. Bürgin-Wolff, H. Gaze, F. Hadziselimovic, et al., “Antigliadin and Antiendomysium Antibody Determination for Coeliac Disease," Archives of Disease in Childhood, Vol. 66, No. 8, 1991, pp. 941-947. doi:10.1136/adc.66.8.941

[11] R. J. Farrell and C. P. Kelly, "Current Concepts: Celiac Sprue,” The New England Journal of Medicine, Vol. 346, 2002, pp. 180-188. doi:10.1056/NEJMra010852

[12] R. Lorini, A. Scaramuzza, L. Vitali, et al., "Clinical Aspects of Coeliac Disease in Children with Insulin-Dependent Diabetes Mellitus,” Journal of Pediatric Endocrinology Metabolism, Vol. 9, No. S, 1996, pp. 101-111. doi:10.1515/JPEM.1996.9.S1.101

[13] M. Markku and C. Pekka, "Celiac Disease," The Lancet, Vol. 349, No. 9067, 1997, pp. 1755-1759.

\section{doi:10.1016/S0140-6736(96)70237-4}

[14] G. R. Corazza, F. Biagi, M. L. Andreani, et al., "Screening Test for Celiac Disease,” The Lancet, Vol. 349, No. 9048, 1997, pp. 325-326. doi:10.1016/S0140-6736(05)62823-1

[15] G. Corrao, G. R. Corazza, M. L. Andreani, et al., "Serological Screening of Celiac Disease: Choosing the Optimal Procedure according to Various Prevalence Values," Gut, Vol. 35, No. 6, 1994, pp. 771-775. doi:10.1136/gut.35.6.771

[16] S. Sulkanen, T. Halttunen, K. Laurila, et al., "Tissue Trans-Glutaminase Autoantibody Enzyme-Linked Imunosorbent Assay in Detecting Celiac Disease,” Gastroenterology, Vol. 115, No. 6, 1998, pp. 1322-1328. doi:10.1016/S0016-5085(98)70008-3

[17] T. Valdimarsson, L. Franzen, E. Grodzinsky, et al., "Is Small Bowel Biopsy Necessary in Adults with Suspected Celiac Disease and Iga Anti-Endomysium Antibodies? $100 \%$ Positive Predictive Value for Celiac Disease in Adults,” Digestive Diseases and Sciences, Vol. 41, No. 1, 1996, pp. 83-87. doi:10.1007/BF02208588

[18] A. Rostom, C. Dube, A. Cranney, et al., "Celiac Disease," Evidence Report/Technology Assessment, No. 104, AHRQ Publication No. 04-E029-2, Rockville, MD: Agency for Healthcare Research and Quality, 2004.

[19] C. Sategna-Guidetti, R. Pulitano, S. Grosso and G. Ferfoglia, "Serum IgA-Antiendomysium Antibody Titers as a Marker of Intestinal Involvement and Diet Compliance in Adult Celiac Sprue,” Journal of Clinical of Gastroenterology, Vol. 17, No. 2, 1993, pp. 123-127. doi:10.1097/00004836-199309000-00007

[20] A. H. Talal, J. A. Murray, J. A. Goeken, et al., "Celiac Disease in Adult Population with Insulin-Dependent Diabetes Mellitus: Use of Endomysial Antibody Testing," The American Journal of Gastroenterology, Vol. 92, No. 8, 1997, pp. 1280-1284.

[21] M. J. Rensch, J. A. Merenich, M. Lieberman, et al., "Gluten-Sensitive Enteropathy in Patients with Insulin-Dependent Diabetes Mellitus," Annals of Internal Medicine, Vol. 124, No. 6, 1996, pp. 564-567.

[22] A. K. Carlsson, I. E. Axelsson, S. K. Borulf, et al., "Prevalence of IgA-Antiendomysium and IgA-Antigliadin Autoantibodies at Diagnosis of Insulin-Dependent Diabetes Mellitus in Swedish Children and Adolescents," Pediatrics, Vol. 103, 1999, pp. 1248-1252. doi:10.1542/peds.103.6.1248

[23] C. Aygun, S. Uraz, T. Damci, Z. Osar, V. Yumuk, E. Akdenizli and H. Ilkova, "Celiac Disease in an Adult Turkish Population with Type I Diabetes Mellitus,” Digestive Diseases and Sciences, Vol. 50, No. 8, 2005, pp. 1462-1466. doi:10.1007/s10620-005-2862-8

[24] M. Hummel, E. Bonifacio, M. Stern, J. Dittler, A. Schimmel and A. G. Ziegler, "Development of Celiac Disease-Associated Antibodies in Offspring of Parents with Type I Diabetes,” Diabetologia, Vol. 43, No. 8, 2000, pp. 1005-1011. doi:10.1007/s001250051483

[25] G. K. T. Holmes, “Long-Term Health Risks for Unrecog- 
nized Coeliac Patients,” In: S. Auricchio and J. K. Visakorpi, Eds., Common Food Intolerance, I, Epidemiology of Coeliac Disease, Karger, Basel, 1992, pp. 105-118.

[26] J. West, R. F. Logan, C. J. Smith, R. B. Hubbard and T. R.
Card, "Malignancy and Mortality in People with Coeliac Disease: Population Based Cohort Study,” British Medical Journal, Vol. 329, No. 7468, 2004, pp. 716-719. 